\title{
Evaluating the Role of Interleukin-36 and Interleukin-38 in Acne Vulgaris Patients
}

F.M.El-Esawy ${ }^{1}$, D.M.Elhabak ${ }^{1}$, S.A.Mohamed ${ }^{2}$ and S.H.Mostafa ${ }^{1}$

${ }^{1}$ Dermatology, Venereology and Andrology Dept., Faculty of Medicine, Benha Univ., Benha, Egypt

${ }^{2}$ Medical Biochemistry and Molecular Biology Dept., Faculty of Medicine, Benha Univ., Benha, Egypt

E-Mail:Sara@gmail.com

\begin{abstract}
Interleukin (IL)- 36 and (IL)- 38 cytokines are individuals from the IL-1 family, which incorporate favorable to infammatory factors and over late years, much has been educated on their significant capacities in the guideline of safe reaction and, particularly, on their function in numerous infammatory skin sicknesses. Be that as it may, until this point, no information have been accounted for on their conceivable inclusion in skin inflammation vulgaris. The point of this examination was to assess the quality articulation levels of Interleukin 36 and IL-38 in skin inflammation vulgaris patients and contrast their levels and solid controls. 100 me mbers were remembered for this investigation Group A : seventy patients with skin inflammation vulgaris of different levels of seriousness. Gathering B: thirty clearly sound people of coordinated age and sex were picked as a benchmark group. Skin inflammation Vulgaris was analyzed and assessed seriousness and movement and research center assessment (IL-36 and IL-38) was finished. IL36 and IL38 mRNA level was fundamentally higher in Correlation
\end{abstract} to seriousness of skin inflammation.

\section{Introduction}

Skin inflammation is a skin condition that happens when the hair follicles become stopped with oil and dead skin cells. It regularly causes whiteheads, clogged pores or pimples, and generally shows up on the face, temple, chest, upper back and shoulders [1].

Skin inflammation is the most widely recognized skin infection of the pilosebaceous unit, and interleukin-1(IL-1) family cytokines along with Th1 and Th17 invulnerable reaction have been characterized as initiators and key entertainers of its pathogenesis [2].

Interleukin-36 (IL-36) sub-relatives are new cytokines of IL-1 family that incorporate IL-36 $\alpha$, IL$36 \beta$, IL-36 $\gamma$, and IL-36 receptor rival (Ra). IL-36 cytokines are predominantly communicated in keratinocytes and monocytes/macrophages and assume a significant function in the tweak of $\mathrm{T}$ assistant (Th) 1 and Th17 invulnerable reactions [3].

Since IL-36 cytokines are mostly communicated in epithelial cells especially in keratinocytes, they have been prevalently concentrated in skin infections. We have recently demonstrated that IL-36 individuals were improved in psoriasis just as in Allergic Contact Dermatitis skin [4].

Moreover, it has been accounted for that an irregularity in IL-1 family agonist and enemy capacities could assume a significant function in cutaneous irritation and in the aggregate of skin harm; specifically, hereditary lack of IL-36Ra may prompt an autoinflammatory condition which basically shows as a serious type of pustular psoriasis [5].

Be that as it may, until this point, IL-36 cytokines have not been concentrated in other two significant provocative skin issues, for example, skin break out and hidradenitis suppurativa (HS) [6].

Subsequently, the point of this primer examination to research the outflow of IL-36 and IL38 sub-relatives in skin break out.
The point of this examination was to assess the quality articulation levels of Interleukin 36 and IL-38 in skin break out vulgaris patients and contrast their levels and sound controls.

\section{Patient and Method}

The Inclusion Criteria for patients were: acne, being over fifteen years of age, not taking any medications for any purposes, and being willing to participate in the study.

\section{Exclusion Criteria}

- Patient less than 15 years old and above 35years.

- Pregnant and lactating women were excluded from both groups.

\section{Administrative design}

This study was approved by the research ethical committee of Banha faculty of medicine .

\section{All participants were divided into two groups}

Group A: seventy patients with acne vulgaris of various degrees of severity.

Group B: thirty apparently healthy individuals of matched age and sex were chosen as a control group.

\subsection{Methods}

Subjects maintained a general diet and were in a stable living condition at least one week before the study and avoided.

\section{Measurement of interleukin 36 and IL-38 in the blood}

Fasting venous blood samples $(3 \mathrm{ml})$ were taken from all studied subjects to asses IL-36 and IL-38 mRNA in all subjects.

Sample was collected in sterile tube and stored at $-20^{\circ} \mathrm{C}$ until analysis. All methods were performed according to the manufacturer's instructions. 
Before taking blood samples a written informed consent was taken from each patient or each subject in the control group.

\section{All patients were subjected to the following}

- A complete History was taken from each patient.

- Complete general Examination to exclude systemic diseases.

- Dermatological clinical examination: Acne Vulgaris can be evaluated according to :

$>$ Onset, course and duration.

$>$ Lesions which may be comedones (whiteheads or blackheads), papules, pustules, nodules or even cyst.

$>$ Site of lesions in face, chest, shoulders and upper back .

$>$ Severity and progression.

$>$ Response to previous treatment,

$>$ Associated with hirsutism and androgenetic alopecia or not.

$>$ Asking female patient about any menstrual disturbence.

- Laboratory examination (IL-36 and IL-38).

This case control study was carried out on seventy adult patients complaining of acne vulgaris, and gender matched healthy volunteers they were selected from those attending the outpatient clinic of dermatology, venereology and andrology department of Banha University Hospital .

\section{Results}

The mean age was 22.48 years. They were 42 males and 58 females (8.7\%).No significant differences were found regarding age and sex between both groups

According to group A (patients with acne vulgaris), the mean age of onset was 17.47 years and $(48.6 \%)$ of patients were with intermittent course and the rest of them were with progressive course. The mean duration time of the disease was 4.63 years ranges from 1 to 14 years

According to previous treatment, 37 (52.9\%) patients treated by topical \& systemic treatment, 18 $(25.7 \%)$ of them received topical treatment while 3 patients $(4.3 \%)$ treated by topical treatment \& chemical peeling , $2(2.9 \%)$ patients received only systemic treatment, one patient received triple therapy (Topical and systemic treatment \& Chemical peeling) and 9 patients $(12.9 \%)$ had no previous treatment.

22 patients (31.4) in group A (patients with acne vulgaris) was association with AGA.

Hirsutism presented in 8 females representing (19.5\%) from

group A female population $(n=41)$.

According to family history in group A (patients with acne vulgaris), $44(62.5 \%)$ patients are with positive family history

According to menstrual history all females in Group B (control) had regular menstrual period while
$16(40 \%)$ female patient in group A had irregular menstrual period.

There was a statically significant difference between the two groups according to menstrual history.

According to severity by GAGS score in group A (patients with acne vulgaris), 21 (30\%) were severe disease, $38(54.3 \%)$ were moderate and $11(15.7 \%)$ were mild.

The following table shows type and distribution of acne. 22 cases suffered from only face acne $(31.4 \%), 8(11.4 \%)$ in Face \& chest, $12(17.1 \%)$ in Face \& back, 27(38.6\%) in Face \& back \& chest and one case $(1.4 \%)$ in Face \& neck.

$41(58.6 \%)$ cases showed scar formation, 24 of them were icepick scar only, 13 were icepick \& rolling, 2 were icepick \& rolling \& boxcar scars and one case of hypertrophic scars. PIH was found in 62(88.6) cases.

IL36 mRNAlevel was significantly higher in group A (patient group) $(2.10 \pm 0.63)$ than Group B (control group) $(1.0 \pm 0.39) .(\mathrm{p}<0.001)$

The diagnostic value for IL36 mRNA level to diagnose acne vulgaris is presented in the following ROC . ROC curve of IL36 mRNA level was conducted for discrimination between cases (group A) and control (group B) as well as severity of disease.

IL36 mRNA level at the best cutoff point $(>1.39)$ predicted patients with acne vulgaris from control with sensitivity $=91.43 \%$ and specificity= $83.33 \%(\mathrm{p}=<0.001)$. AUC succeeded to discriminate between cases and control groups (0.948), positive predictive value was $92.8 \%$, negative predictive value was $80.6 \%$.

IL36 mRNA level was significantly higher in Correlation to severity of acne.

According to relation between severity with IL36 mRNA level in vulgaris group, IL36 mRNA level was significantly higher in severe subgroup than moderate and mild subgroup $(\mathrm{p}=0.003, \mathrm{p}<0.001)$. Also IL36 mRNA level was significantly higher in Moderate subgroup than mild subgroup $(\mathrm{p}=0.003)$.

In mild cases IL36 mRNA level at the best cutoff point $(\leq 1.72)$ predicted patients with acne vulgaris with sensitivity $=81.82 \%$ and specificity $=79.66 \%(\mathrm{p}$ $=<0.001)$. AUC succeeded to discriminate mild cases $(0.876)$, positive predictive value was $42.9 \%$, negative predictive value was $95.9 \%$.

In severe cases IL36 mRNA level at the best cutoff point $(>2.05)$ was with sensitivity $=71.43 \%$ and specificity $=69.39 \%(\mathrm{p}=<0.001)$, , positive predictive value was $50.0 \%$, negative predictive value was $85.0 \%$.

There were statically significant differences in IL36 mRNA level according to severity (conducted from ROC curves) in mild, moderate and severe cases. 
The following tables $(15,16)$ shows relation between IL36 mRNA level and some different parameters in acne vulgaris group.

IL36 mRNA level was significantly higher in relation to Duration of disease, previous treatment, hirsutism, association with AGA, distribution in the body and scar formation

There were statically no significant differences in IL36 mRNA level according to (Age, sex, age of onset of acne, course, family history, menstrual history, scar type and PIH)

IL38 mRNAlevel was significantly higher in group A (patient group) $(0.92 \pm 0.45)$ than Group B (control group) $(0.52 \pm 0.22) .(\mathrm{p}<0.001)$

The diagnostic value for IL38 mRNA level to diagnose acne vulgaris is presented in the following ROC . ROC curve of IL38 mRNA level was conducted for discrimination between cases (group A) and control (group B) as well as severity of disease.

IL38 mRNA level at the best cutoff point $(>0.6)$ predicted patients with acne vulgaris from control with sensitivity $=80.0 \%$ and specificity $=76.67 \%(\mathrm{p}=$ $<0.001)$. AUC succeeded to discriminate between cases and control groups (0.810), positive predictive value was $88.9 \%$, negative predictive value was $62.2 \%$.

IL38 mRNA level was significantly higher in Correlation to severity of acne. $(r=0.720, p<0.001)$

According to relation between severity with IL38 mRNA level in vulgaris group, IL38 mRNA level was significantly higher in severe subgroup than moderate and mild subgroup $(\mathrm{p}<0.001, \mathrm{p}<0.001)$. Also IL36 mRNA level was significantly higher in Moderate subgroup than mild subgroup $(\mathrm{p}=0.002)$.

In mild cases IL38 mRNA level at the best cutoff point $(\leq 0.68)$ predicted patients with acne vulgaris with sensitivity $=90.91 \%$ and specificity $=77.97 \%(\mathrm{p}$ $=<0.001)$. AUC succeeded to discriminate mild cases (0.876), positive predictive value was $43.5 \%$, negative predictive value was $97.9 \%$

In severe cases IL38 mRNA level at the best cutoff point $(>0.96)$ was with sensitivity $=80.95 \%$ and specificity $=71.43 \%(\mathrm{p}=<0.001)$, , positive predictive value was $54.8 \%$, negative predictive value was $89.7 \%$.

IL38 mRNA level was significantly higher according to severity (conducted from ROC curves) in mild, moderate and severe cases.

The following tables $(24,25)$ shows relation between IL38 mRNA level and some different parameters in acne vulgaris group. IL38 mRNA levels were significantly higher according to (Duration of disease, previous treatment, hirsutism, association with AGA, distribution in the body, scar formation and $\mathrm{PIH}$ )

There were statically no significant differences in IL38 mRNA level according to (Age, sex, age of onset of acne, course, family history, menstrual history and scar type).

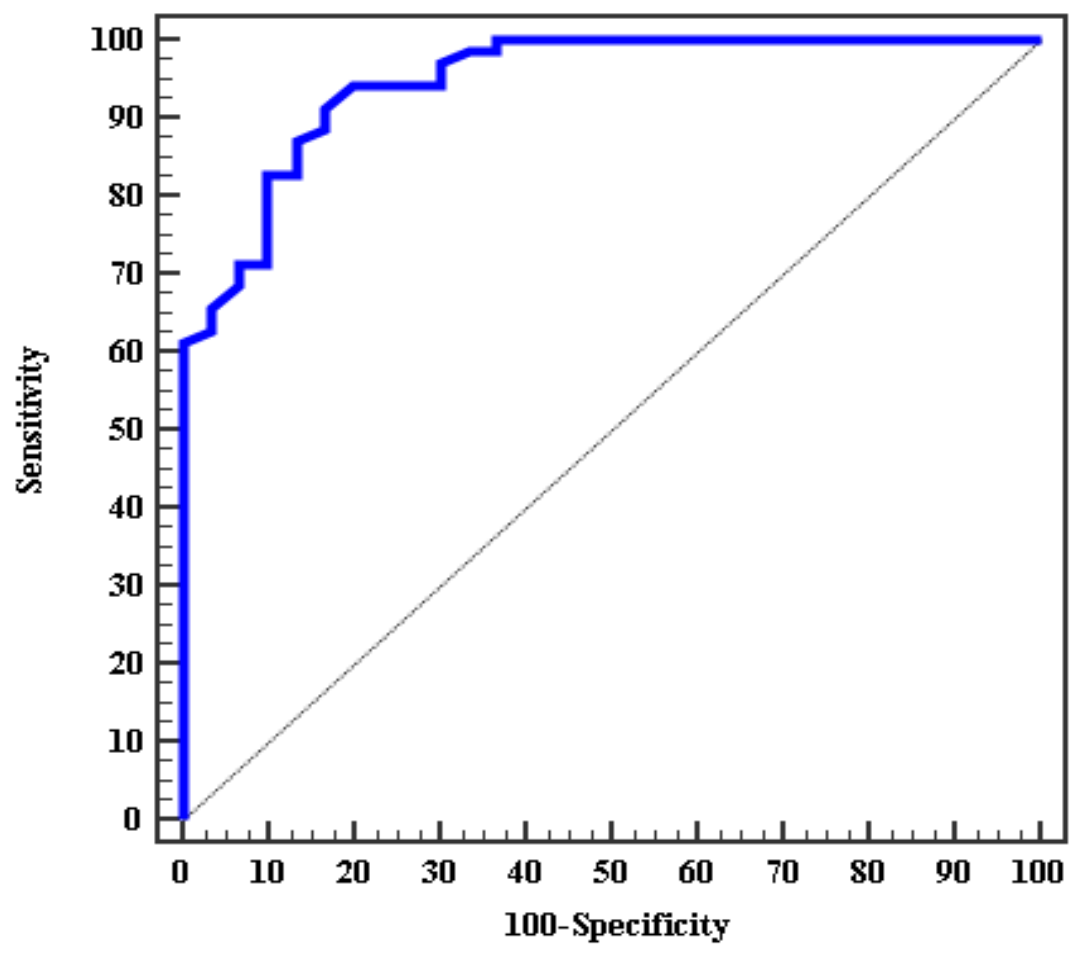

Fig (1) ROC curve for IL36 mRNA level to diagnose acne vulgaris cases ( $n=70 / 100)$ 


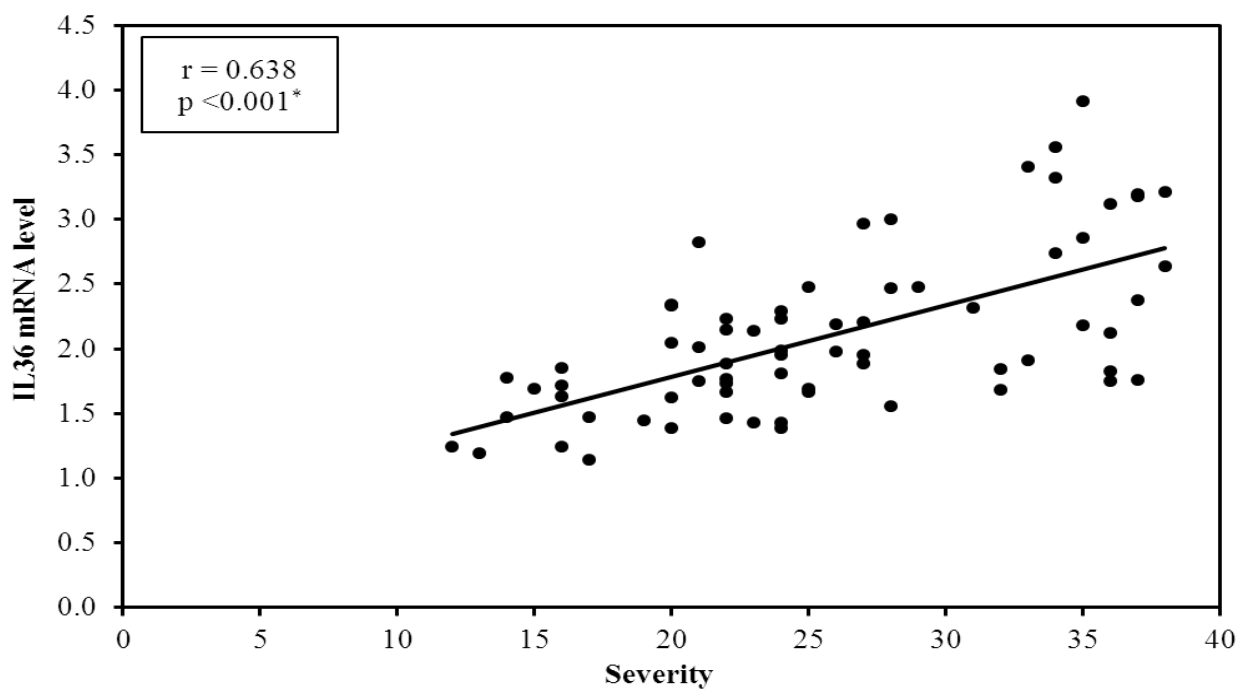

Fig (2) Correlation between severitywith IL36 mRNA levelin vulgaris group $(n=70)$.

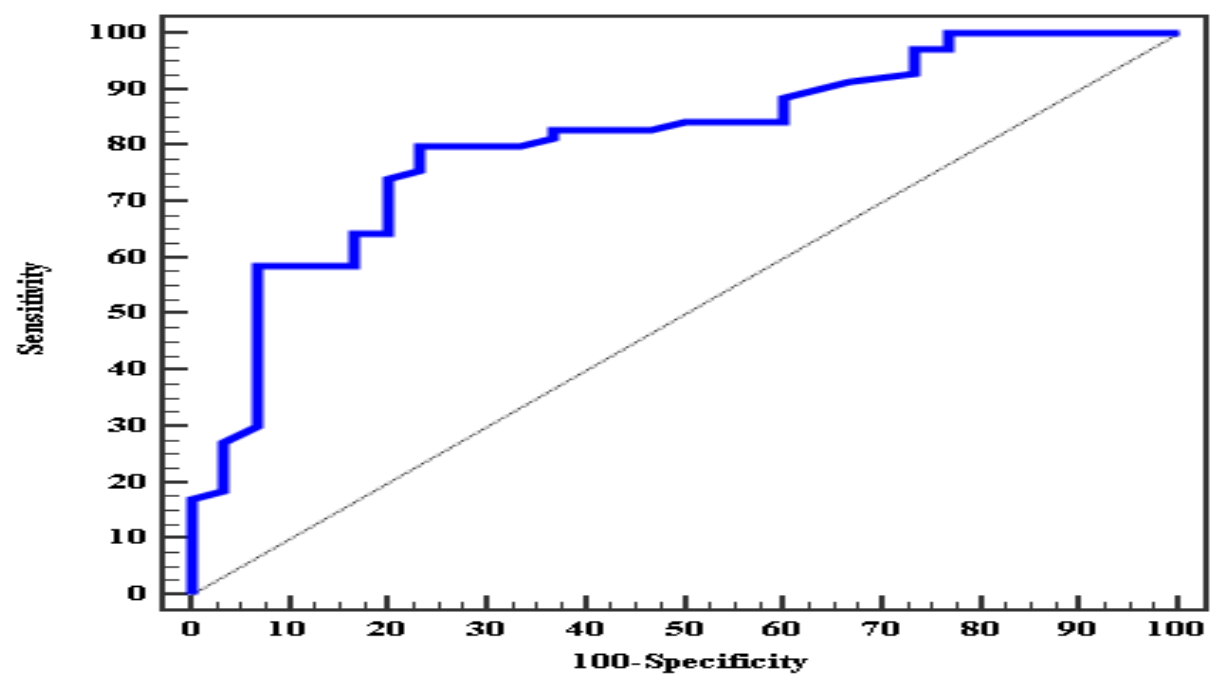

Fig (3) ROC curve for IL38 mRNA level to diagnose acne vulgaris cases ( $n=70 / 100)$.

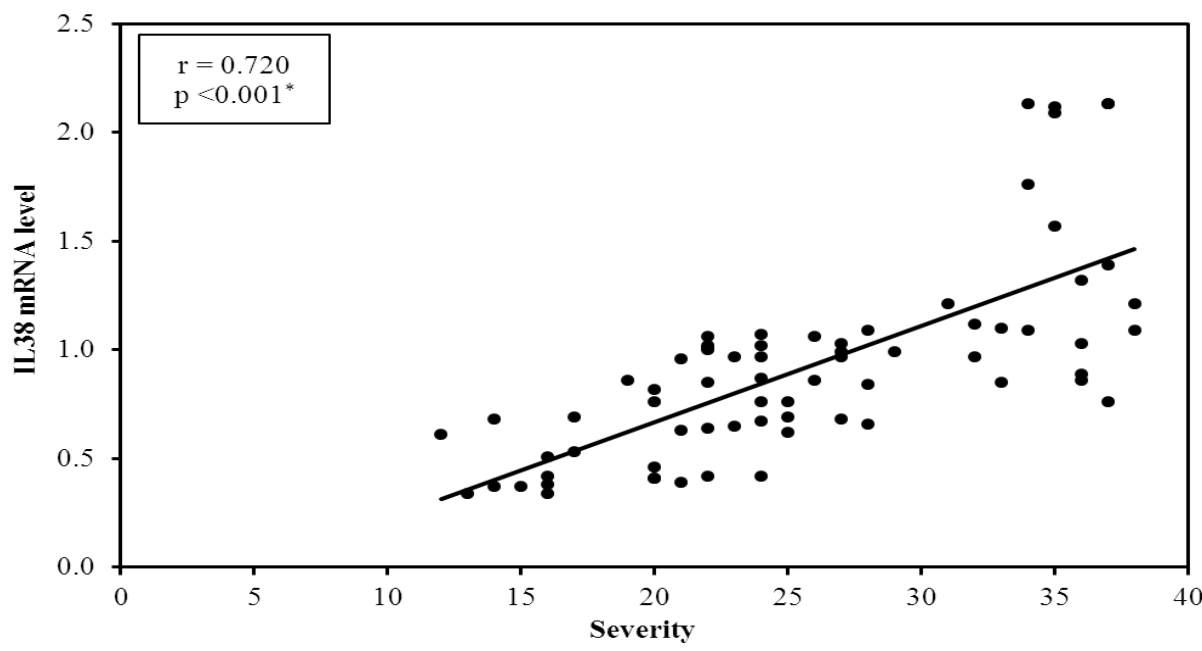

Fig (4) Correlation between severity with IL38 mRNA levelin vulgaris group $(n=70)$. 


\section{Discussion}

Interleukin (IL)- 36 sub-relatives are new cytokines of IL- 1 family that incorporate IL-36 $\alpha$, IL$36 \beta$, IL-36 $\gamma$, and IL-36 receptor rival (Ra). IL-36 cytokines are mostly communicated in keratinocytes and monocytes/macrophages [7] and assume a significant function in the tweak of $\mathrm{T}$ partner (Th) 1 and Th17 insusceptible reactions [8].

IL-36Ra ties IL-36R and blocks the enlistment of the second receptor IL-1 receptor frill protein (RAcP), going about as receptor opponent [9].

Since IL-36 cytokines are basically communicated in epithelial cells, especially in keratinocytes, they have been prevalently concentrated in skin sicknesses. We have recently indicated that IL-36 individuals were upgraded in psoriasis just as in ACD skin B [1].

What's more, it has been accounted for that an awkwardness in IL-1 family agonist and opponent capacities could assume a significant function in cutaneous infammation and in the aggregate of skin harm; specifically, hereditary defciency of IL-36Ra may prompt an autoinfammatory condition which essentially shows as a serious type of pustular psoriasis [10].

In any case, until now, IL-36 cytokines have not been concentrated in other significant infammatory skin problems, for example, skin break out. Skin inflammation vulgaris is the most widely recognized skin sickness of the pilosebaceous unit, and IL-1 family cytokines along with Th1 and Th17 safe reactions have been defned as initiators and key entertainers of its pathogenesis [2]. Our information demonstrated the association of IL-36 and IL-38 in skin break out vulgaris.

The significance of IL-36 cytokines in a few infammatory skin issues has just been accounted for [1], however information exhibiting their association in skin break out are so far missing. The arrival of supportive of infammatory arbiters, for example, tumor rot factor (TNF)- $\alpha$ and IL-17, and the follicular impediment, intervened by infundibular keratosis and hyperplasia of the follicular epithelium [11], are the primary highlights shared by skin inflammation and speak to pathogenetic joins between this infection. In spite of the fact that they are both clinically described by pustules, they are for the most part not set off by contamination [11].

Skin inflammation originats in the follicular infundibulum and are portrayed by follicular impediment prompts dilatation and arrival of keratin and microbes into the dermis, instigating an incredible infammatory reaction primarily Th1 and Th17 interceded. Without a doubt, it has been accounted for that IL-36 $\gamma$ transgenic mice which overexpress IL-36 $\gamma$ in basal keratinocytes show acanthosis and hyperkeratosis of the skin [12].

\section{Conclussion}

From the results of present study, it is concluded that both IL36mRNA and IL38mRNA might play arole in the pathogenesis of AV. Moreover, they are associated with more severe grades of AV.

\section{References}

[1] A.Balato, M.Mattii, G.Caiazzo, A.Raimondo,

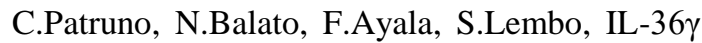
is involved in psoriasis and allergic contact dermatitis.J Invest Dermatol,Vol.136, PP.15201523,2016.

[2] E.Ingham, E.A . Eady, C.E. Goodwin, J.H. Cove, W.J. Cunliffe, Pro-inflammatory levels of interleukin-1 alpha-like bioactivity are present in the majority of open comedones in acne vulgaris. J Invest Dermatol,Vol.98, PP.895-901,1992.

[3] A.Balato, M.Schiattarella, S.Lembo, M.Mattii, N.Prevete, N.Balato, F.Ayala, Interleukin-1 family members are enhanced in psoriasis and suppressed by vitamin D and retinoic acid.Arch Dermatol Res,Vol.305, PP.255-262,2013.

[4] S. Z. Chong, K.W. Tan, F.H. Wong, Y.L. Chua, Y.Tang, V.Angeli, D.M. andKemeny, CD8 T cells regulate allergic contact dermatitis by modulating CCR2-dependent TNF/iNOSexpressing Ly6C + CD11b+ monocytic cells.J Invest Dermatol ,Vol.134, PP.666-676,2014.

[5] G.Ganzetti, A.Campanati, E.Molinelli, A. Offidani, Biologic therapy in inflammatory and immunomediated skin diseases: safety profile.Curr Drug Saf;Vol.11,PP.12-21,2016.

[6] M.S.Gresnigt, D.E.van, F.L.Veerdonk, Biology of IL-36 cytokines and their role in disease.SeminImmunol,Vol.25, PP.458-465, 2013.

[7] M.S. Gresnigt, F.L. van de Veerdonk ,Biology of IL-36 cytokines and their role in disease. Semin Immunol ,Vol.25, PP.458-465, 2013.

[8] J.E. Towne, B.R. Renshaw, J .Douangpanya, B.P. Lipsky, M. Shen, C.A. Gabel, J.E. Sims Interleukin-36 (IL-36) ligands require processing for full agonist (IL-36alpha, IL-36beta, and IL36gamma) or antagonist (IL-36Ra) activity. J Biol Chem,Vol.286, PP.42594-42602, 2011.

[9] R. Thomi, M. Kakeda, N. Yawalkar, C. Schlapbach, R.E .Hunger, Increased expression of the interleukin-36 cytokines in lesions of hidradenitis suppurativa. J Eur Acad Dermatol Venereol, Vol.35(50), PP.15-25, 2017.

[10] K. Sugiura, A .Takemoto, M. Yamaguchi, H. Takahashi, Y. Shoda, T. Mitsuma, K .Tsuda, E .Nishida, Y. Togawa, K .Nakajima, A. Sakakibara, S .Kawachi, M .Shimizu, Y .Ito, T .Takeichi, M .Kono, Y .Ogawa, Y .Muro, A .Ishida-Yamamoto, S .Sano, H .Matsue, A .Morita, H .Mizutani, H .Iizuka, M. Muto, M. Akiyama, The majority of generalized pustular 
psoriasis without psoriasis vulgaris is caused by defciency of interleukin-36 receptor antagonist. J Invest Dermatol,Vol.133, PP.2514-2521, 2013.

[11]E .Prens, I. Deckers , Pathophysiology of hidradenitis suppurativa: an update. J Am Acad Dermatol ,Vol.73, PP. 8-11,2015.
[12] A .Grant, T .Gonzalez, M.O. Montgomery, V .Cardenas, F.A. Kerdel ,Infiximab therapy for patients with moderate to severe hidradenitis suppurativa: a randomized, double-blind, placebocontrolled crossover trial. J Am Acad Dermatol ,Vol.62, PP.205-217, 2010. 